\title{
Reaction behavior modeling of metal hydride based on FeTiMn using numerical simulations
}

\author{
Mario Bedrunka ${ }^{1,2}$, Nils Bornemann ${ }^{2}$, Gerd Steinebach ${ }^{1}$, Dirk Reith $^{1,3}$ \\ Grantham-Allee 20, 53757 Sankt Augustin
}

\begin{abstract}
In this contribution, we perform computer simulations to expedite the development of hydrogen storages based on metal hydride. These simulations enable in-depth analysis of the processes within the systems which otherwise could not be achieved. That is, because the determination of crucial process properties require measurement instruments in the setup which are currently not available. Therefore, we investigate the reliability of reaction values that are determined by a design of experiments.
\end{abstract}

Specifically, we first explain our model setup in detail. We define the mathematical terms to obtain insights into the thermal processes and reaction kinetics. We then compare the simulated results to measurements of a 5 -gram sample consisting of iron-titanium-manganese (FeTiMn) to obtain the values with the highest agreement with the experimental data. In addition, we improve the model by replacing the commonly used Van't-Hoff equation by a mathematical expression of the pressure-composition-isotherms (PCI) to calculate the equilibrium pressure.

Finally, the parameters' accuracy is checked in yet another with an exist-

\footnotetext{
${ }^{1}$ Institute of Technology, Resource and Energy-Efficient Engineering, Bonn-Rhein-Sieg University of Applied Sciences, Grantham-Allee 20, 53757 Sankt Augustin, Germany

${ }^{2}$ GKN Sinter Metals Engineering GmbH, Krebsoege 10, 42477 Radevormwald, Germany

${ }^{3}$ Fraunhofer-Institute for Algorithms and Scientific Computing (SCAI), Schloss Birlinghoven, 53757 Sankt Augustin, Germany
}

Preprint submitted to Journal of Elsevier

January 18, 2021 
ing metal hydride system. The simulated results demonstrate high concordance with experimental data, which advocate the usage of approximated kinetic reaction properties by a design of experiments for further design studies. Furthermore, we are able to determine process parameters like the entropy and enthalpy.

Keywords: Metal hydride storage, simulations

2010 MSC: 00-01, 99-00

\section{Introduction}

Water is not only indispensable for every form of life, its elements hydrogen and oxygen will shape the future, when energy supply becomes more and more critical due to the global population growth [1].

Therefore, the challenge is to store this huge amount of hydrogen, whereas oxygen is already available in the air. The state of the art mainly provides three different technical solutions: Pressure tanks store the hydrogen at a great pressure level, hence they require high safety standards; liquid storages require extremely low temperatures, which makes it inefficient; last, solid-state storages contain the hydrogen in chemical form. Latter have a high storage density of hydrogen at a low pressure compared to pressure tanks, at the price of large weights. Due to the properties of the systems, the storage systems have different field of applications. Depending on their operating conditions, the storages are selected.

Despite the relevance of all technical solutions, this study focuses on solidstate storages and investigates the reaction behavior of hydrogen and a solidstate alloy. Recent studies [2] 3] 4] already dealt with solid-state hydrogen storages based on metal hydride and their simulations. The purpose of these studies was to achieve in-depth understanding of the processes inside metal hydride solids. These studies provide thermodynamic and kinetic reaction coefficients 
for several metal hydride materials. For example, conventional values are available for a basic iron-titanium alloy $[5]$ 6. However, the kinetic reaction properties of iron-titanium-manganese (FeTiMn) utilized by this research have not yet been sufficiently studied in the literature. There is no precedent literature available for our alloy or similar additives. Although these coefficients are essential to simulate the reaction behavior between metal hydride based on this iron-titanium-manganese alloy and hydrogen. This is why the conventional values have to be checked.

This study provides mathematical principles of the reaction processes between metal hydride and hydrogen and describes the physical processes inside a characteristic storage system. Hence, the development of the charge level can 35 be calculated considering the movement of gaseous hydrogen, thermal conduction inside the metal hydride solid as well as the system's pressure level. This leads to a simulation model which allows to analyze several design concepts of solid-state storages regarding various metal hydrides. The model requires to be verified to assess the accuracy of the simulation. Therefore, data collected in the laboratory is compared to simulation results. However, the crucial kinetic reaction parameters cannot be determined due to not available measurement instruments. This is why a design of experiments is used: Kinetic reaction coefficients are approximated by comparing them to experimental data and references of a basic FeTi alloy. The reliability of this process is investigated. 


\section{Mathematical model}

This paragraph provides a description of the mathematical model. Previous projects [2] 3] [4] dealt with the reproduction of the reaction behavior of metal hydrides with a mathematical model. Conservation laws describe the mass distribution (i.e. hydrogen density; metal hydride density) and energy (i.e. temperature) distribution inside the tank system. An equation considers these state variables and calculates the reaction rate between hydrogen and metal hydride. This contribution assumes the fundamental model and refines it.

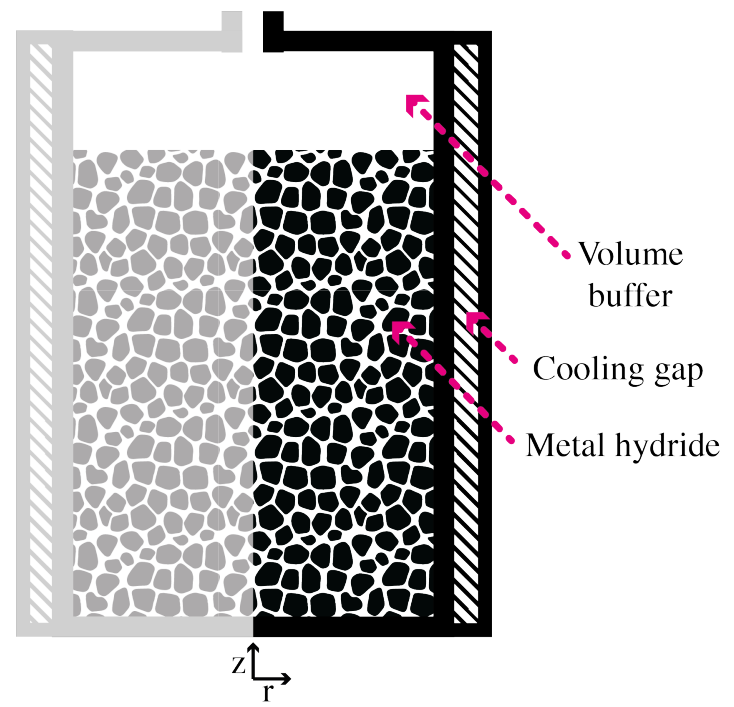

Figure 1: Illustration of the model structure. The simulation model consists of three different areas: metal hydride solid (porous media); volume buffer (empty area) and the heat exchanger (lined area).

Model structure. The model considers 3 areas: The metal hydride solid; the volume buffer and the heat exchanger filled with water. Figure 1 illustrates the model's structure. The metal hydride area is presented by a homogeneous solid. A volume buffer has to be taken into consideration for design concepts because of the volume expansion during the absorption. The tank's cylindrical geometry favors a simplification: Instead of observing a 3 dimensional volume, 
the tank is represented by a two-dimensional numerical grid in radial $\mathrm{r}$ and axial z direction (i.e. metal hydride solid; volume buffer). However, the annular gap is represented by a one-dimensional numerical grid in axial direction. It is assumed that variation of state variables in radial direction are negligible within the annular gap. Depending on the areas the model considers the following state variables:

Metal hydride solid:

- $\rho_{\mathrm{H}_{2}}$ - Hydrogen density

- $\rho_{\mathrm{MH}}$ - Metal hydride solid density

- $T_{\mathrm{MH}}$ - Metal hydride solid temperature

Volume buffer:

- $\rho_{\mathrm{H}_{2}}$ - Hydrogen density

- $T_{\mathrm{H}_{2}}$ - Hydrogen temperature

Heat exchanger (gap):

- $T_{\mathrm{H}_{2} \mathrm{O}}$ - Water temperature 
Conservation of energy. The model considers the solid's temperature $T_{\mathrm{MH}}$, the temperature within the volume buffer $T_{\mathrm{H}_{2}}$ and the water's temperature $T_{\mathrm{H}_{2} \mathrm{O}}$. Conservation equations of energy calculate the temperature changes of these mediums. Regarding the cylindrical geometry of the pressure tank the equations may be written as follows [2] [3] [7]:

$$
\begin{array}{rlrl}
\left(\rho c_{\mathrm{P}}\right)_{\mathrm{EFF}} \frac{\partial T_{\mathrm{MH}}}{\partial t} & = & \lambda_{\mathrm{EFF}}\left(\frac{1}{r} \frac{\partial T_{\mathrm{MH}}}{\partial r}+\frac{\partial^{2} T_{\mathrm{MH}}}{\partial r^{2}}+\frac{\partial^{2} T_{\mathrm{MH}}}{\partial z^{2}}\right)-\dot{m}_{\mathrm{R}} \Delta H+\dot{Q}_{\mathrm{MH}-\mathrm{H}_{2} \mathrm{O}} \\
\left(\rho c_{\mathrm{P}}\right)_{\mathrm{H}_{2}} \frac{\partial T_{\mathrm{H}_{2}}}{\partial t}= & \lambda_{\mathrm{H}_{2}}\left(\frac{1}{r} \frac{\partial T_{\mathrm{H}_{2}}}{\partial r}+\frac{\partial^{2} T_{\mathrm{H}_{2}}}{\partial r^{2}}+\frac{\partial^{2} T_{\mathrm{H}_{2}}}{\partial z^{2}}\right)+\dot{Q}_{\mathrm{H}_{2}-\mathrm{H}_{2} \mathrm{O}} \\
\left(\rho c_{\mathrm{P}}\right)_{\mathrm{H}_{2} \mathrm{O}} \frac{\partial T_{\mathrm{H}_{2} \mathrm{O}}}{\partial t}= & \lambda_{\mathrm{H}_{2} \mathrm{O}} \frac{\partial^{2} T_{\mathrm{H}_{2} \mathrm{O}}}{\partial z^{2}}-u_{\mathrm{H}_{2} \mathrm{O}}\left(\rho c_{\mathrm{P}}\right)_{\mathrm{H}_{2} \mathrm{O}} \frac{\partial T_{\mathrm{H}_{2} \mathrm{O}}}{\partial z}-\dot{Q}_{\mathrm{MH}_{-} \mathrm{H}_{2} \mathrm{O}}
\end{array}
$$

The first terms on the right side describe the heat conduction inside the medium, whereby $\lambda$ is the thermal conductivity of each medium. Using cylinder coordinates $r$ defines the radial direction and $z$ the axial direction. The amount of heat which is produced or absorbed inside the solid is related to the amount of hydrogen, which is charged $\dot{m}_{\mathrm{ABS}}^{\mathrm{R}}$ and/ or $\dot{m}_{\mathrm{DES}}^{\mathrm{R}}$ discharged per unit time, and is represented by the middle term on the right side of Equation 1, whereby $\Delta H$ describes the enthalpy of the reaction; $\rho$ is the density; and $c_{p}$ the heat capacity. The middle term on the right side of Equation 3 takes the water flux inside the heat exchangers into account. For the calculation of the temperature change inside the solid, both factors define effective values, considering the behavior of hydrogen and metal hydride $[3]$ :

$$
\begin{aligned}
\left(\rho c_{\mathrm{P}}\right)_{\mathrm{EFF}} & =\left(\rho c_{\mathrm{P}}\right)_{\mathrm{MH}}+\epsilon\left(\rho c_{\mathrm{P}}\right)_{\mathrm{H}_{2}} \\
\lambda_{\mathrm{EFF}} & =\lambda_{\mathrm{MH}}+\epsilon \lambda_{\mathrm{H}_{2}}
\end{aligned}
$$

$\dot{Q}$ defines the heat flux between the heat exchanger's medium and the solid or hydrogen inside the storage. The heat flux depends on the temperature gradient $\Delta T$ between the mediums, the exchange area $A$, in this case the tank surface, and the heat transition coefficient $U[9]$ : 


$$
\dot{Q}=U A \overbrace{\left(T_{\mathrm{H}_{2} \mathrm{O}}-T_{\mathrm{MH}} / \mathrm{H}_{2}\right)}^{\Delta T}
$$

Conservation of mass. A conservation equation of mass calculates the hydrogen distribution considering processes such as the reaction and the gas transfer. This equation may be written in general as follows [2] [3]:

$$
\epsilon \frac{\partial \rho_{\mathrm{H}_{2}}}{\partial t}=-\nabla\left(\rho_{\mathrm{H}_{2}} u_{\mathrm{H}_{2}}\right)-\dot{m}
$$

whereby $\dot{m}$ represents sources and/ or consumption of hydrogen (i.e. reaction rate $\dot{m}^{\mathrm{R}}$ between the solid and hydrogen or the flow through the valve of the pressure tank) and $\epsilon$ the porosity of the metal hydride. The first term on the right side of the Equation 7 describes the gas transfer through the porous solid. The equation is transformed by regarding the cylindrical geometry of the pressure tank:

$$
\epsilon \frac{\partial \rho_{\mathrm{H}_{2}}}{\partial t}=-\frac{1}{r} \frac{\partial}{\partial r}\left(r \rho_{\mathrm{H}_{2}} u_{\mathrm{H}_{2}}^{r}\right)-\frac{\partial}{\partial z}\left(\rho_{\mathrm{H}_{2}} u_{\mathrm{H}_{2}}^{z}\right)-\dot{m}
$$

The gas velocity $u$ is mainly influenced by a pressure difference and is obtained from the Darcy equation. The Darcy theorem represents the flow through a porous medium and is written as follows [7] 10] 11]:

$$
u_{\mathrm{H}_{2}}=-\frac{\kappa}{\mu} \nabla p
$$

Assuming that hydrogen is an ideal gas with $p=\rho T R m_{\mathrm{MOL}}^{-1}$. $R$ defines the universal gas constant and $m_{\mathrm{MOL}}$ the molar mass of hydrogen, gives:

$$
\epsilon \frac{\partial \rho_{\mathrm{H}_{2}}}{\partial t}=\frac{\kappa}{\mu} \frac{R}{m_{\mathrm{MOL}}} \frac{1}{r} \frac{\partial}{\partial r}\left(r \rho_{\mathrm{H}_{2}} \frac{\partial \rho_{\mathrm{H}_{2}} T_{\mathrm{H}_{2}}}{\partial r}\right)+\frac{\partial}{\partial z}\left(\rho_{\mathrm{H}_{2}} \frac{\partial \rho_{\mathrm{H}_{2}} T_{\mathrm{H}_{2}}}{\partial z}\right)-\dot{m}
$$

with the dynamic viscosity $\mu$. The Konzeny-Carman equation is used to determine the permeability $\kappa$ as follows with the diameter of the used powder ${ }_{110} \quad d_{p}[13$. 


$$
\kappa=\frac{\epsilon^{2} d_{\mathrm{P}}}{150(1-\epsilon)^{2}}
$$

The conservation of mass also describes the development of metal hydride density $\rho_{\mathrm{MH}}$. This development is only influenced by the reaction rate $\dot{m}_{\mathrm{R}}$ between the metal hydride solid and the hydrogen [2]:

$$
\frac{\partial \rho_{\mathrm{MH}}}{\partial t}=\dot{m}_{\mathrm{R}}
$$

Reaction rate. The core of the metal hydride simulation is based on the calculation of the reaction kinetics for absorption (ABS) and desorption (DES). The mathematical term takes process variables like the temperature, the pressure as well as the mass concentration into account to calculate the reaction rate $\dot{m}_{\mathrm{R}}$ [2]:

$$
\begin{gathered}
\dot{m}_{\mathrm{ABS}}^{\mathrm{R}}=k \exp \left(\frac{E}{R T_{\mathrm{MH}}}\right) \cdot \frac{p_{e q}-p}{p_{e q}} \cdot\left(\rho_{\mathrm{MH}}^{\mathrm{MAX}}-\rho_{\mathrm{MH}}\right) \\
\dot{m}_{\mathrm{DES}}^{\mathrm{R}}=k \exp \left(\frac{E}{R T_{\mathrm{MH}}}\right) \cdot \underbrace{\frac{p_{e q}-p}{p_{e q}}}_{f_{2}} \cdot \underbrace{\left(\rho_{\mathrm{MH}}-\rho_{\mathrm{MH}}^{\mathrm{MIN}}\right)}_{f_{3}}
\end{gathered}
$$

The first term describes the reaction behavior of the metal hydride alloy used and takes into consideration that the activation energy $E$ has to be overcome by the reaction. The reaction coefficient $k$ defines the ability of the metal to absorb the hydrogen. The relation between the pressure level $p$ inside the pressure tank and the equilibrium pressure $p_{e q}$ is described by the second term. The greater the difference between these values, the more hydrogen is charged or discharged. M.Ron [14 describes different ways to describe this relationship. The last term calculates the difference between the loading status and the equilibrium state of stored hydrogen [15]. $\rho_{\text {MAX }}$ and $\rho_{\text {MIN }}$ are constant values or defined by an approximated function. Figure 2 illustrates the relation of the involved variables. For example, two operation points are marked. Depending 
on the current state of charge and pressure the relation of the discussed terms are illustrated according to the PCI.

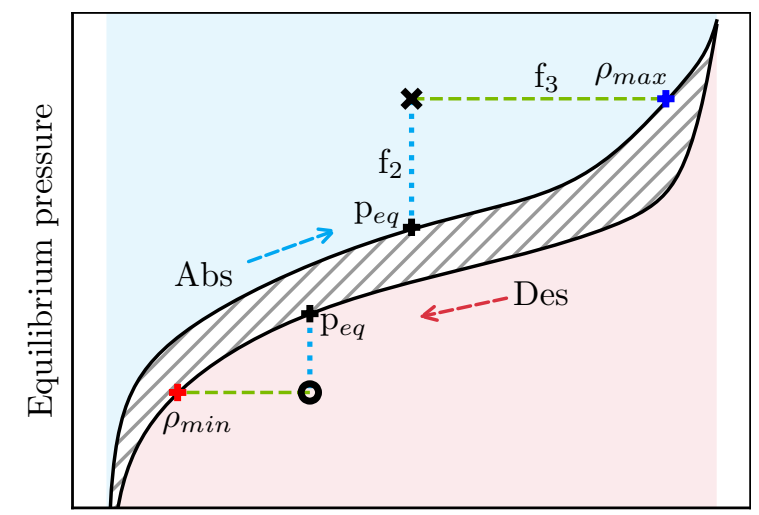

State of charge

Figure 2: Illustration of the involved variables of the reaction equation by means of a pressurecomposition-isotherm. Red area: Desorption; blue area: Absorption.

The partial differential equations must be completed by initial and boundary conditions: At the beginning of the simulation, the metal hydride's temperature and density, the pressure of the gas and the temperature of the water assumed to be homogeneous [3]. Therefore, the initial conditions are chosen as follows.:

$$
\begin{array}{ll}
\text { Temperatures: } & T_{\mathrm{MH}}(r, z, t=0)=T_{\mathrm{MH}}^{0} \\
& T_{\mathrm{H}_{2}}(r, z, t=0)=T_{\mathrm{H}_{2}}^{0} \\
& T_{\mathrm{H}_{2} \mathrm{O}}(z, t=0)=T_{\mathrm{H}_{2} \mathrm{O}}^{0}
\end{array}
$$

$$
\begin{array}{ll}
\text { Densities: } & \rho_{\mathrm{MH}}(r, z, t=0)=\rho_{\mathrm{MH}}^{0} \\
& \rho_{\mathrm{H}_{2}}(r, z, t=0)=\rho_{\mathrm{H}_{2}}^{0}
\end{array}
$$

The flow temperature of the water at the inlet to give values for $T_{i n}(t)$ is set. At the edge of the metal hydride bed it is assumed that there is no temperature change. Therefore, the boundary conditions are as follows, where 
$z=0$ describes the bottom of the pressure Tank, $z=Z$ the top, $r=0$ the axis of the cylindrical pressure tank and $r=R$ the surface of the pressure tank [3]:

$$
\begin{aligned}
& \text { Temperatures: } T_{\mathrm{H}_{2} \mathrm{O}}(z, t)=T_{\text {in }}(t) \text { at } z=0 \\
& \frac{\partial T_{\mathrm{MH}}}{\partial z}=\quad 0 \text { at } z=0 \\
& \frac{\partial T_{\mathrm{H}_{2}}}{\partial z}=\quad 0 \text { at } z=Z \\
& \frac{\partial T_{\mathrm{MH}}}{\partial r}=\quad 0 \text { at } r=0 ; r=R \\
& \frac{\partial T_{\mathrm{H}_{2}}}{\partial r}=\quad 0 \text { at } r=0 ; r=R \\
& \text { Densities: } \quad \frac{\partial \rho_{\mathrm{H}_{2}}}{\partial z}=\quad 0 \text { at } z=0 ; z=Z \\
& \frac{\partial \rho_{\mathrm{H}_{2}}}{\partial r}=\quad 0 \text { at } r=0 ; r=R
\end{aligned}
$$

Numerical methods. The defined mathematical models are partial differential equations (PDE). Matlab provides well-engineered tools to solve ordinary differential equations (ODE). These equation solvers require a conversion of the PDEs into ODEs by the method of lines. After a discretization in space the local derivatives are approximated by finite differences [16. This leads to a large system of ODEs. The differential equations system is then solved by taking the solver ode15s from Matlab [17. 


\section{Iron-titanium-manganese alloy}

The material used for the model validation consists of an iron-titanium-

150

for a temperature. In other words, the amount of hydrogen stored in metal hy-

dride for a given pressure and temperature after a long term. This characteristic

pressure is known as equilibrium pressure and influences the formation of metal hydride and therefore the reaction behavior.

165

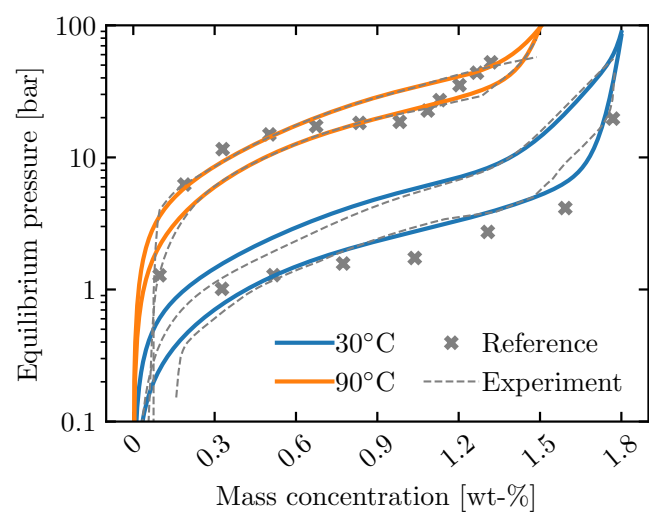

Figure 3: Measured PCIs of FeTiMn based on courtesy of GKN. Reference is taken from H. Buchner [19] that is given for $25^{\circ} \mathrm{C}$ and $90^{\circ} \mathrm{C}$ 
A hysteresis is shown between the absorption and desorption. The hysteresis can be explained through the conducted work during the absorption, since the lattice expansion requires more energy, whereas during the desorption the lattice is already expanded and the hydrogen atoms escape freely. Therefore, the pressure plateau is always higher during absorption than desorption. With increasing temperature, the lattice expansion grows. The required energy decreases and therefore the hysteresis does too. H. Buchner [19] provides PCI data for $25^{\circ} \mathrm{C}$ and $90^{\circ} \mathrm{C}$. The characteristic of the measured PCI curves correlate with the reference data in the literature. The small gap in the comparison of the lower temperature can be explained due to the temperature difference of the reference data used.

Pressure-composition-isotherms - mathematical model. The equilibrium pressure $p_{e q}$ is essential to calculate the reaction rate of metal hydrides (Equation 13 14). Previous studies [13 [1] 2] used the Van't-Hoff equation to determine the equilibrium pressure at any given state assuming an ideal PCI: The pressure plateaus have no gradient. However, this idealized assumption results in a calculation error, which is shown in 20 .

Hence, Herbrig et al. 20] have developed a mathematical model to represent PCIs in an accurate way. This model is based on exponential functions, which include a series expansion. The respective terms are weighted by adjusted coefficients $\alpha$ and $\beta$ to express the PCI characteristic of an individual material alloy. The advantage of this model is that the equilibrium pressure can be determined at any given temperature $T$ in kelvin and concentration $w$ in weight-percentage.

It has to be said, that this equation has not any physical explanation but shows high accordance with the measured data.

$$
\begin{array}{rc}
p_{e q}=\frac{10 w}{1+10 w} & \left(\exp \left(\alpha_{0} T^{-1}+\alpha_{1} T+\alpha_{2} T^{2}+\alpha_{3} w+\alpha_{4} w^{2}+\alpha_{5} w^{3}\right)\right. \\
+ & \exp \left(\beta_{0} T+\beta_{1} T^{2}+\beta_{2} T w+\beta_{3} w+\beta_{4} w^{2}\right) \\
+ & \left.\exp \left(50\left(w-\beta_{5}\right)\right)\right)
\end{array}
$$


The coefficients $\alpha$ and $\beta$ are obtained by means of a Matlab curving fitting tool (cftool). This tool provides capabilities to approximate measured data points by polynomials, interpolation or custom equations. The adjusted coefficients are shown in Table 1. Compared to Herbrig et al. [20] this study uses a slightly modified series expansion, which fits better the material's PCI measured. The PCI-model is more accurate than using the Van't-Hoff equation, which is already shown in 20]. The Van't-Hoff equation disregards the state of charge. Hence, only one equilibrium pressure is calculated for a given temperature. This approach is acceptable for an ideal PCI with a constant pressure plateau, but not for the material used in this study. Figure 3 shows the described curve model for FeTiMn using adjusted coefficients and compares the model to experimental data.

Table 1: Adjusted coefficients of the mathematical PCI curve model for FeTiMn

\begin{tabular}{crllrrrl}
\hline Coefficient & Absorption & Desorption & Coefficient & Absorption & Desorption \\
\hline$\alpha_{0}$ & -2793 & $/$ & -2506 & $\beta_{0}$ & -0.08377 & $/$ & -0.1186 \\
$\alpha_{1}$ & 0.05211 & $/$ & 0.0329 & $\beta_{1}$ & $8.617 \mathrm{e}-5$ & $/$ & $3.193 \mathrm{e}-4$ \\
$\alpha_{2}$ & $-7.184 \mathrm{e}-5$ & $/$ & $-3.011 \mathrm{e}-5$ & $\beta_{2}$ & 0.04009 & $/$ & 0.01629 \\
$\alpha_{3}$ & 2.3760 & $/$ & 4.124 & $\beta_{3}$ & 5.804 & $/$ & -20.42 \\
$\alpha_{4}$ & -0.3568 & $/$ & -2.964 & $\beta_{4}$ & -3.313 & $/$ & 11.89 \\
$\alpha_{5}$ & -0.2035 & $/$ & 0.961 & $\beta_{5}$ & 1.735 & $/$ & 1.74 \\
\hline
\end{tabular}


Van't-Hoff isochores - enthalpy and entropy. Van't-Hoff isochores describe the

state of equilibrium of any chemical reaction. In case of metal hydrides this state of equilibrium is expressed by the correlation between the equilibrium pressure and the temperature. Therefore, these isochores can be determined due to the measured PCI. The Van't-Hoff equation demonstrates the relationship between these parameters and requires further variables such as the enthalpy and entropy.

$$
\log \left(\frac{p_{e q}}{p_{0}}\right)=\frac{\Delta H}{R T}+\frac{-\Delta S}{R},
$$

$\Delta H$ defines the enthalpy; $R$ the universal gas constant; $\Delta S$ the entropy; $p_{e q}$ the equilibrium pressure; $p_{0}$ the atmospheric pressure and $T$ the temperature. With the aid of value pairs $\left(T, p_{e q}\right)$ given from the measured PCI, the isochores can be approximated for both absorption and desorption. The Van't-Hoff isochroes are approximated using the PCIs to investigate the entropy $\Delta S$ and enthalpy

$\Delta H$ of FeTiMn. The entropy can be defined as $\Delta S=-109.88 \mathrm{~J} \mathrm{~K}^{-1}$ whereas the enthalpy changes due to the sloping PCI. The determined enthalpy for both absorption and desorption can be expressed by Equation 17 and Equation 18 in the range between $0.2 \mathrm{wt}-\%$ and $1.3 \mathrm{wt}-\%$ depending on the state of charge. Otherwise, the limit value of the boundaries $(0.2 \mathrm{wt}-\% ; 1.3 \mathrm{wt}-\%)$ is used.

$$
\begin{aligned}
& \Delta H_{\mathrm{ABS}}=-2327 w^{2}+8424 w-35230 \\
& \Delta H_{\mathrm{DES}}=-2668 w^{2}+8500 w-36510
\end{aligned}
$$

\section{Model validation}

The calculated results are validated by a comparison with measurements: First, the simulation of a heating-cooling process verifies the thermal diffusion inside the metal hydride's solid; then, a design of experiments approximates kinetic reaction coefficients to reproduce the reaction kinetics; finally, a comparison with data of an existing system checks the validity of the calibrated values. 


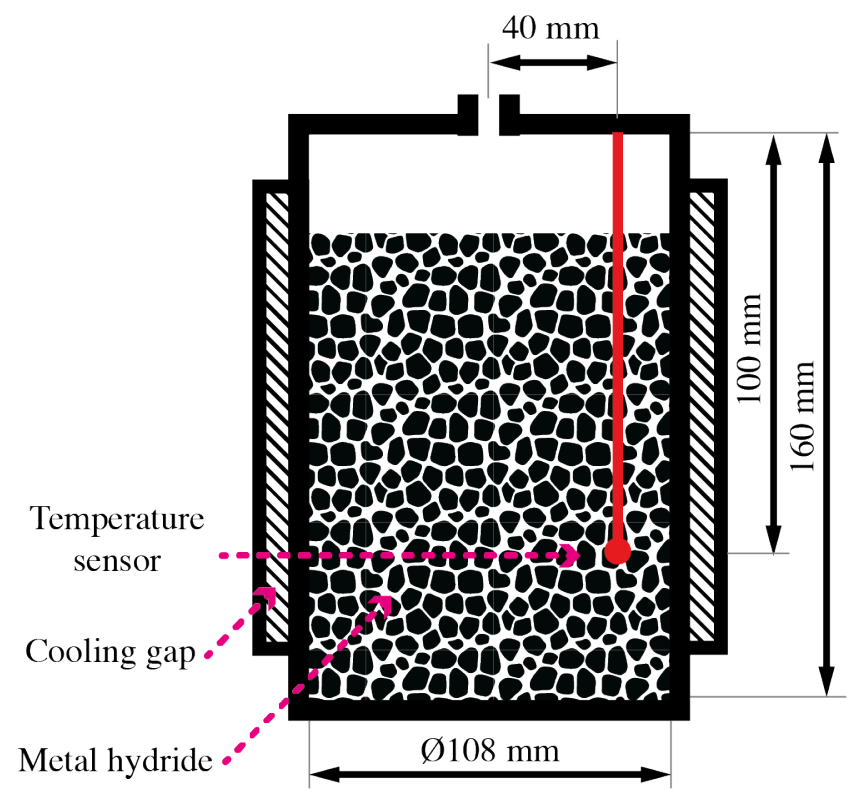

Figure 4: Tankdesign used for the thermal validation that is filled with metal hydride (porous media) and provides a volume buffer. An annular gap surrounds the pressure tank as a heat exchanger (lined). The temperature can be measured due to a sensor within the solid (red dot)

Temperature validation. A pressure tank used for the validation is illustrated in Figure 4. The pressure tank is filled with FeTiMn described and is evacuated to avoid endothermic or exothermic reaction effects. An annular gap surrounds the pressure tank as a heat exchangers. Then, water inside this gap influences the thermal activity of the solid and allows a control of system. One temperature sensor is placed inside the metal hydride storage to measure the temperature process which is compared to the simulation results. The used properties are shown in Table 2. The thermal parameters $\lambda_{\mathrm{MH}}$ and $c_{\mathrm{P}}^{\mathrm{MH}}$ are measured by GKN.

The comparative data describes a heating-cooling process. It is assumed that the initial bed temperature is homogeneous. The measured water temper- 
Table 2: Summary of the material properties used for FeTiMn which are the input parameters of the simulation.

\begin{tabular}{lcrl}
\hline Properties & Symbol & Value & Unit \\
\hline Thermal conductivity & $\lambda_{\mathrm{MH}}$ & $6.2-5$ & $\mathrm{~W} \mathrm{~m}^{-1} \mathrm{~K}^{-1}$ \\
& & $25^{\circ} \mathrm{C}-100{ }^{\circ} \mathrm{C}$ & \\
& $\lambda_{\mathrm{H}_{2}}$ & 0.1805 & $\mathrm{~W} \mathrm{~m}^{-1} \mathrm{~K}^{-1}$ \\
& $\lambda_{\mathrm{H}_{2} \mathrm{O}}$ & 0.6 & $\mathrm{~W} \mathrm{~m}^{-1} \mathrm{~K}^{-1}$ \\
& $\Delta H_{\mathrm{ABS}}$ & $-33638--28211$ & $\mathrm{~J}$ \\
Enthalpy & $\Delta H_{\mathrm{DES}}$ & $-34917--29969$ & $\mathrm{~J}$ \\
& $\Delta S$ & -109.88 & $\mathrm{~J} \mathrm{~K}^{-1}$ \\
Entropy & $\Delta_{\mathrm{P}}$ & 682.4 & $\mathrm{~J} \mathrm{~kg}^{-1} \mathrm{~K}^{-1}$ \\
Heat capacity & $c_{\mathrm{P}}^{\mathrm{MH}}$ & 14304 & $\mathrm{~J} \mathrm{~kg}^{-1} \mathrm{~K}^{-1}$ \\
& $c_{\mathrm{P}}^{\mathrm{H}}$ & 4182 & $\mathrm{~J} \mathrm{~kg}^{-1} \mathrm{~K}^{-1}$ \\
& $c_{\mathrm{P}}^{\mathrm{H}_{2} \mathrm{O}}$ & &
\end{tabular}

ature of the inlet is approximated and is set as the data input for the simulation. Figure 5 illustrates the simulation results compared to the measured temperatures. It can be demonstrated that the characteristic form of the simulated temperature process resembles the measured temperature. Thus the validation of the temperature conductivity is proven. 


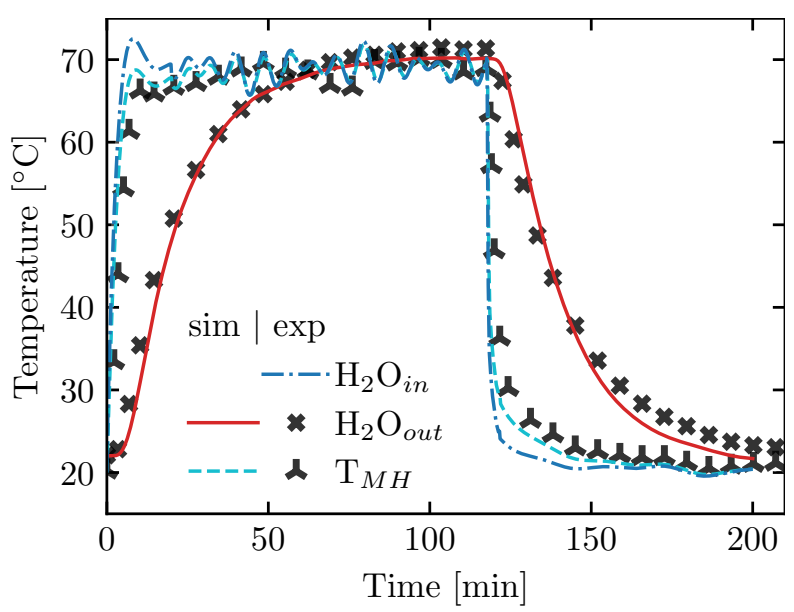

Figure 5: Validation of a thermal conduction process inside a metal hydride storage. The thermal activity is influenced by the input temperature of the water inside the heat exchangers. The validation of the thermal conduction is consisting of a heating-up and a cooling-down process. exp descripes the measured data and calc the simulated results.

Reaction validation. A high-pressure weighting station 'STA HP1' from the company Linseis is utilized to measure the storage capacity. The set-up is illustrated in Figure 6. This measurement aperture bases upon an electromagnetic beam scale with a measurement precision of $0.1 \mu \mathrm{g}$ and is designed for hydrogen experiments. The measured weight gain of metal hydride results in the mass concentration and therefore the hydrogen capacity achieved. The pressure tank has a total volume of $2 \mathrm{~L}$. This allows an accurate dosage of pressure. A $5 \mathrm{~g}$ sample of metal hydride is in a case, which is located on the temperature sensor. The heating element in form of a spiral surrounds the case. The cooling occurs due to convection.

The determination of crucial process properties require further measurement 255 instruments in the setup which are currently not available. This is why we utilize a design of experiments to approximate the reaction coefficient $k$ for both desorption and absorption. The values of the activation energy are taken from the basic alloy FeTi: [5] $E_{a b s}=23800 \mathrm{~J} \mathrm{~mol}^{-1}$ and $E_{\text {des }}=19870 \mathrm{~J} \mathrm{~mol}^{-1}$. 


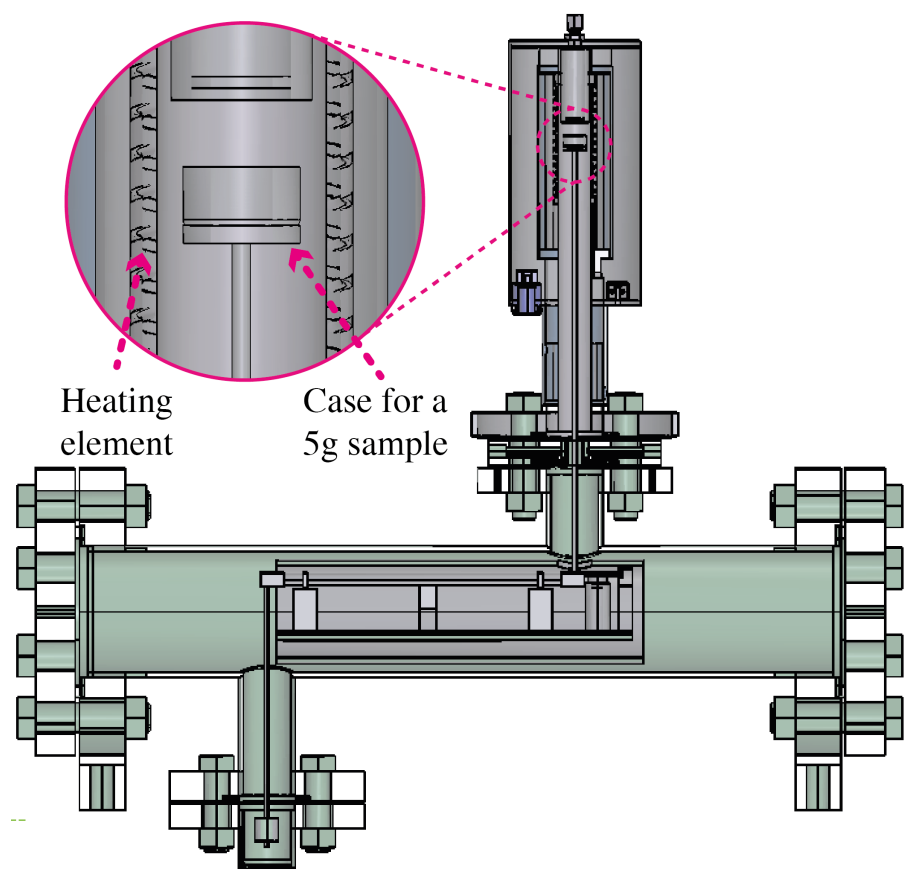

Figure 6: Illustration of the high-pressure weighting station used to measure the hydrogen capacity of FeTiMn. The measurement aperture bases upon an electromagnetic beam scale and is designed for hydrogen experiments from the company Linseis. A metal hydride sample is fixed in a case. A heating element surrounds the case and controls the sample's temperature. Then, the weight gain is measured.

The aim of this approach is the reproduction of the stored hydrogen of several experiments (i.e. 15 bar and $30^{\circ} \mathrm{C} ; 40$ bar and $30^{\circ} \mathrm{C} ; 40$ bar and $40{ }^{\circ} \mathrm{C}$ ). The reaction coefficient is varied in the range of 1 to 100. A comparison operator $R^{2}$ rates the accordance with the measurements. This operator is calculated as follows [21]:

$$
R^{2}=\frac{\sum_{i=1}^{n_{r}}\left(\hat{y}_{i}-\bar{y}\right)^{2}}{\sum_{i=1}^{n_{r}}\left(y_{i}-\bar{y}\right)^{2}}
$$

The factor $R^{2}$ indicates the extent of variability of the dependent parameter, whereby $n_{r}$ defines the quantity of data; $y_{i}$ defines the experimental data; $\hat{y}_{i}$ 
defines the simulation data and $\bar{y}$ is the average of the simulated results. The operator $R^{2}$ takes a value of one if the simulated results correspondent to the experiment; or deviates from one if the simulated results differ from the experiment. Figure 7 shows the averaged values of the comparison operator for the reaction coefficients of both absorption and desorption.

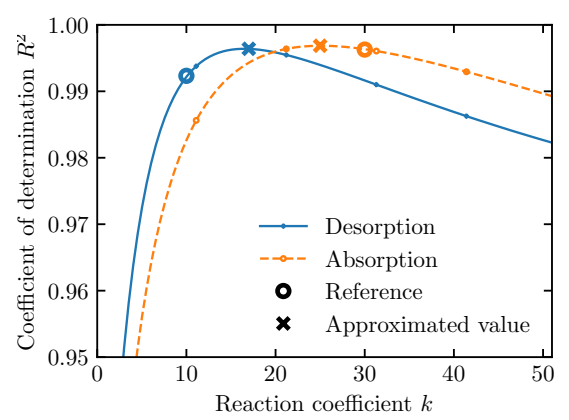

Figure 7: Illustration of the values of comparison operator of the DoE in order to calibrate the reaction coefficient. The value that demonstrate the highest accordance to the experimental data are marked with ' $\mathrm{x}$ '.

The simulated results with the best comparison operator, demonstrate high concordance with the experiments, which can be shown in Figure 8. 6] Matsushita et al. give values of reaction coefficients for a conventional FeTi alloy. These values are marked in Figure 7. As shown, the approximated values are similar to those of Matsushita et al., which also result in high accuracy. Thus, the striven values of the reaction coefficients $\left(k_{a b s}=25 k_{\text {des }}=17\right)$ are sufficiently approximated. The reaction kinetics is reproduced accurately for each experiment. However, a difference in hydrogen capacity is achieved, for the case of $40 \mathrm{bar}$ and $30^{\circ} \mathrm{C}$ which results from an insufficient modeling of the PCI at the boundary.

Transfer validation. Data from GKN's pilot project of a residential home is 285 given (Knappenhaus in south Tirol - www.hy2green.de). A metal hydride stor- 

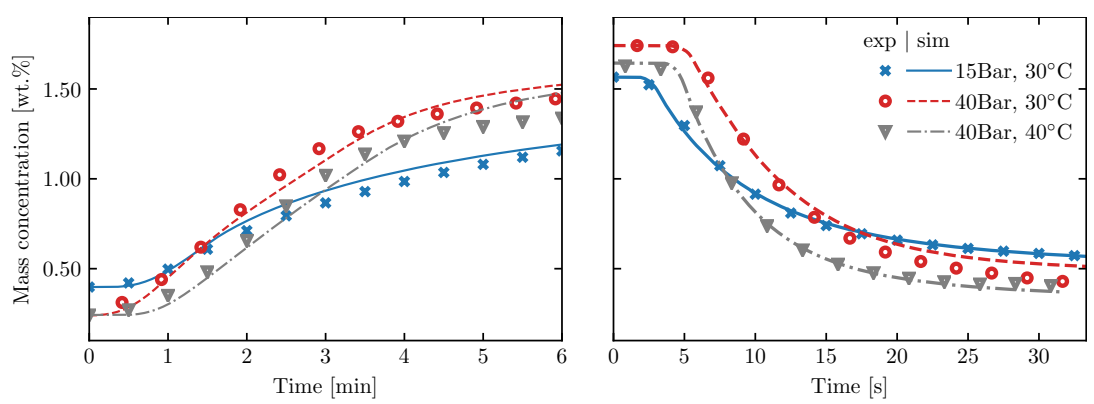

Figure 8: Illustration of the computed results (calculation) compared to the measured data (exp) using the values of kinetic reaction properties with the best comparison operator $R^{2}$. The measured data is shown by an average with its standard deviation. Left: Absorption; Right: Desorption; A: exp. 15 bar and $30^{\circ} \mathrm{C} ;$ B: exp. 40 bar and $30^{\circ} \mathrm{C} ; C$ : exp. 40 bar and $40^{\circ} \mathrm{C}$
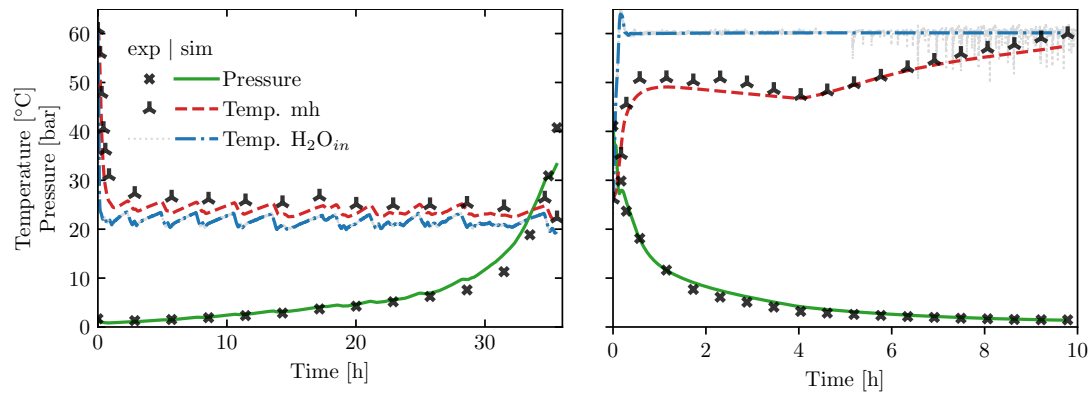

Figure 9: Illustration of the comparison of the simulated and the measured results of the GKN's Knappenhaus. Left: Absorption process, where the supplied mass flow of hydrogen is $6.6 \mathrm{~L} \mathrm{~min}{ }^{-1}$. Right: Desorption process, where the released mass flow of hydrogen is $-36.5 \mathrm{~L} \mathrm{~min}{ }^{-1}$ for the first $4 \mathrm{~h}$ and is then decreased. The mass flows as well as the water temperature are smoothed by mathematical terms. 


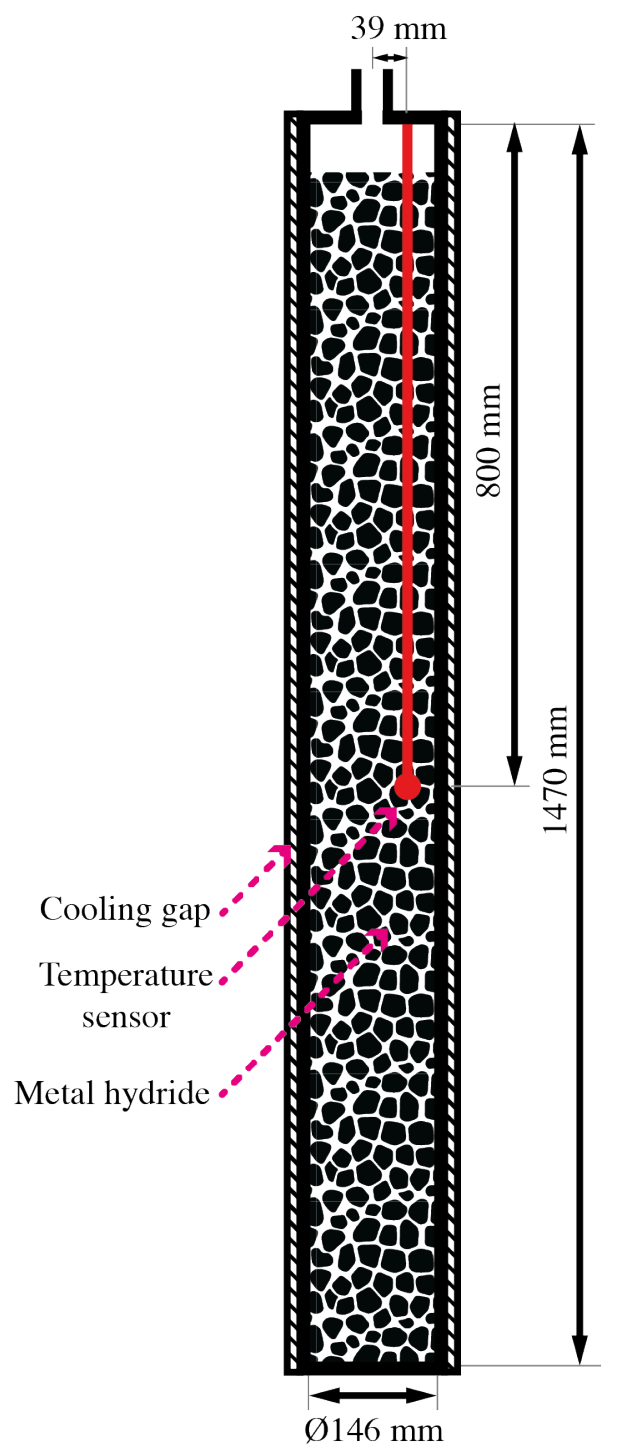

Figure 10: Tankdesign of Knappenhaus used for the transfer validation that is filled with metal hydride (porous media) and provides a volume buffer. An annular gap surrounds the pressure tank as a heat exchanger (lined). The temperature can be measured due to a sensor within the solid (red dot). 
age system is already implemented, where the pressure tank is filled with $79.8 \mathrm{~kg}$ of FeTiMn. Instead of measuring the mass of the metal hydride to draw conclusions about the mass concentration, the current hydrogen capacity can only be estimated using the pressure level.

\section{Conclusion}

310

The present study investigates solid-state hydrogen storage systems based on metal hydrides such as an iron-titanium-manganese alloy (FeTiMn). A mathematical model is utilized to analysis the reaction behavior of this material. The aim is to obtain insights and an understanding of the processes inside the system components such as the pressure tank or the heat exchanger. 
This study provides an equation which fits the measured pressure-compositionisotherms (PCIs) of FeTiMn. Therefore, the equilibrium pressure can be calculated for any working temperature and state of charge. The simulation model requires thermodynamic properties of FeTiMn which have not been studied in available publications and therefore require to be determined. In this contribution Van't-Hoff isochores are deduced by using measured PCIs of FeTiMn to maintain the entropy and enthalpy of formation. These isochores represent the equilibrium pressures which influence the formation of metal hydride. The Van't-Hoff equation defines these isochores and involves the entropy $\Delta S$ as well as the enthalpy $\Delta H$. With the aid of a fitting tool the unknown properties are calibrated $\left(\Delta S=-109.88 \mathrm{~J} \mathrm{~K}^{-1}, \Delta H_{\mathrm{ABS}}^{\text {avg. }}=-29748 \mathrm{~J} \mathrm{~mol}^{-1}\right.$; $\left.\Delta H_{\mathrm{DES}}^{\mathrm{avg} .}=-31212 \mathrm{~J} \mathrm{~mol}^{-1}\right)$.

Further reaction parameters are approximated by a design of experiments because there was no access to measurement equipment. The reaction coefficients are altered for several simulation cycles and then assessed by comparing to experimental data. The simulations show high concordance with the measurements given by an real metal hydride storage system $\left(k_{a b s}=25 k_{d e s}=17\right)$. This supports the reliability of approximated parameters by a DoE for tank modeling and to achieve an understanding of the occurred processes.

The next steps are to investigate the usage of metal hydride storages in real applications. Question arises if it is possible to use metal hydride storages to supply enough energy in form of hydrogen. These questions are discussed in our following paper, which is actual in preparation 22].

\section{Acknowledgement}

This research was supported by GKN Sinter Metals GmbH. We thank our colleagues Bettina Neumann, Thomas Schupp, Dr. Markus Schneider and 
Markus Laux for helpful discussions and Dominik Wilde for comments that greatly improved the manuscript.

\section{References}

\section{References}

[1] World population prospects - population division. Population/

¿ [2] S. S. Mohammadshahi, E. M. A. Gray, C. J. Webb, A review of mathe-

a matical modelling of metal-hydride systems for hydrogen storage applications, International Journal of Hydrogen Energy 41 (5) (2016) 3470-3484. doi:10.1016/j.ijhydene.2015.12.079. URL http://dx.doi.org/10.1016/j.ijhydene.2015.12.079

[3] P. Muthukumar, S. V. Ramana, Numerical simulation of coupled heat and mass transfer in metal hydride-based hydrogen storage reactor, Journal of Alloys and Compounds 472 (1-2) (2009) 466-472. doi:10.1016/j. jallcom.2008.04.088.

${ }_{360}^{\text {口 }}$ [4] M. Valizadeh, M. Aghajani Delavar, M. Farhadi, Numerical simulation of a heat and mass transfer during hydrogen desorption in metal hydride storage tank by Lattice Boltzmann method, International Journal of Hydrogen Energy 41 (1) (2016) 413-424. doi:10.1016/j.ijhydene.2015.11.075.

U. URL https://linkinghub.elsevier.com/retrieve/pii/ S0360319915026877

[5] H. Chung, J.-Y. Lee, Hydriding and dehydriding reaction rate of feti intermetallic compound, International journal of Hydrogen energy 10 (7-8) (1985) 537-542. 
[6] M. MATSUshitA, M. I. andIkko TAJIMA, M. ABE, H. TOKUYAMA,

[ [7] absorption in a metal-hydrogen reactor, International Journal of Hydrogen Energy 20 (1) (1995) 43-52. doi:10.1016/0360-3199(93)E0007-8.

¿ [8] L. Dinachandran, G. Mohan, Numerical simulation of the parametric

1 influence on the wall strain distribution of vertically placed metal hydride based hydrogen storage container, International Journal of Hydrogen Energy 40 (16) (2015) 5689-5700. doi:10.1016/j.ijhydene.2015.02.067. URL https://linkinghub.elsevier.com/retrieve/pii/ S036031991500422X

[9] R. Marek, K. Nitsche, Praxis der Wärmeübertragung, Carl Hanser Verlag GmbH \& Co. KG, München, 2015. doi:10.3139/9783446445529. 9783446445529

[10] S. NASRALLAH, Heat and mass transfer models in metal-hydrogen reactor, International Journal of Hydrogen Energy 22 (1) (1997) 67-76. doi:10.1016/S0360-3199(96)00039-0 S0360319996000390

[11] S. N. Nyamsi, F. Yang, Z. Zhang, An optimization study on a the finned tube heat exchanger used in hydride hydrogen storage system - analytical method and numerical simulation, International Journal of Hydrogen Energy 37 (21) (2012) 16078-16092. doi:10.1016/j.ijhydene.2012.08.074. 
URL

https://linkinghub.elsevier.com/retrieve/pii/ S0360319912018769

[12] A. T. Wijayanta, K. Nakaso, T. Aoki, Y. Kitazato, J. Fukai, Effect 400 of pressure, composition and temperature characteristics on thermal response and overall reaction rates in a metal hydride tank, International Journal of Hydrogen Energy 36 (5) (2011) 3529-3536. doi:10.1016/j.ijhydene.2010.12.047. URL https://linkinghub.elsevier.com/retrieve/pii/ S036031991002389X

[13] B. J. Hardy, D. L. Anton, Hierarchical methodology for modeling hydrogen storage systems. Part I: Scoping models, International Journal of Hydrogen Energy 34 (5) (2009) 2269-2277. doi:10.1016/j.ijhydene.2008.12.070

[1] URL https://linkinghub.elsevier.com/retrieve/pii/ S0360319908017722

[14] M. Ron, The normalized pressure dependence method for the evaluation of kinetic rates of metal hydride formation/decomposition Journal of Alloys and Compounds 283 (1-2) (1999) 178-191. doi:10.1016/S0925-8388(98)00859-7. S0925838898008597

[15] W. Luo, K. J. Gross, A kinetics model of hydrogen absorption and desorption in ti-doped naalh4, Journal of Alloys and Compounds 385 (1-2) (2004) 224-231.

[16] W. Dahmen, A. Reusken, Numerik für Ingenieure und Naturwissenschaftler, Springer-Lehrbuch, Springer Berlin Heidelberg, Berlin, Heidelberg, 2008. doi:10.1007/978-3-540-76493-9.

URL http://link.springer .com/10.1007/978-3-540-76493-9

[17] L. F. Shampine, M. W. Reichelt, The matlab ode suite, SIAM journal on scientific computing 18 (1) (1997) 1-22. 
[18] R. Wakabayashi, S. Sasaki, N. Okinaka, T. Akiyama, et al., Self-ignition combustion synthesis of tife1- xmnx hydrogen storage alloy, International journal of hydrogen energy 34 (22) (2009) 9122-9127.

[19] H. Buchner, Energiespeicherung in Metallhydriden, Springer-Verlag, 2013.

${ }_{430}$ [20] K. Herbrig, L. Röntzsch, C. Pohlmann, T. Weißgärber, B. Kieback, Hydrogen storage systems based on hydride-graphite composites: Computer simulation and experimental validation, International Journal of Hydrogen Energy 38 (17) (2013) 7026-7036. doi:10.1016/j.ijhydene.2013.03.104.

[21] K. Siebertz, D. van Bebber, T. Hochkirchen, Statistische Versuchspla435 nung, Springer Berlin Heidelberg, Berlin, Heidelberg, 2017. doi:10.1007/ 978-3-662-55743-3.

URL http://link. springer.com/10.1007/978-3-662-55743-3

[22] M. Bedrunka, N. Bornemann, G. Steinebach, D. Reith, A Metal Hydride System for a Forklift: Feasibility Study on Portable Chemical Storage of ${ }_{440}$ Hydrogen Using Numerical Simulation, Submitted to International Journal of Hydrogen Energy (2021). 